\title{
Quantifying “normalized" regional left ventricular contractile function in ischemic coronary artery disease
}

\author{
Matthew C. Henn, MD, ${ }^{\mathrm{a}}$ Brian P. Cupps, PhD, ${ }^{\mathrm{a}}$ Julia Kar, PhD, ${ }^{\mathrm{a}}$ Kevin Kulshrestha, ${ }^{\mathrm{a}}$ Danielle Koerner, ${ }^{\mathrm{a}}$ \\ Alan C. Braverman, MD, ${ }^{b}$ and Michael K. Pasque, MD $^{a}$
}

\begin{abstract}
Objective: When significant coronary lesions are identified by angiography, regional left ventricular (LV) contractile function often plays a role in determining candidacy for revascularization. To improve on current subjective and nonquantitative metrics of regional LV function, we tested a z-score "normalization" of regional strain information quantified from clinically acquired high-resolution LV geometric datasets.
\end{abstract}

Methods: Test subjects $(\mathrm{n}=120)$ underwent cardiac MRI with multiple 3-dimensional strain parameters calculated from tissue tag-plane displacement data. Sixty healthy volunteers contributed strain parameter data at each of $15,300 \mathrm{LV}$ grid points, to form a normal human strain database. Point-specific database comparisons were made in 60 patients who had documented coronary artery disease (CAD), by angiography. Patient-specific, color-coded 3-dimensional LV maps of z-score-normalized contractile function were generated.

Results: Blinded clinical review indicated that $55 \%$ (33 of 60 ) of the patients with CAD had significant regional contractile abnormalities by 1 of 3 "gold-standard" criteria: (1) Q waves on electrocardiography (ECG); (2) infarct on radionuclide single-photon emission computed tomography (SPECT); or (3) akinesia or dyskinesia on echocardiography. Consistency among all gold-standard metrics was found for only $19 \%$ ( 6 of 31 ) of patients with CAD who had $\geq 2$ available metrics. Blinded MRI-based, multiparametric, strain z-score localization of contractile abnormalities was accurate in 89\% (ECG), 97\% (SPECT), and 95\% (echocardiography).

Conclusions: Nonsubjective normalization of regional LV contractile function by z-score calculation from a normal human strain database can localize and quantitatively display regional wall motion abnormalities in patients with CAD. This high-resolution localization of regional wall motion abnormalities may help improve the accuracy of therapeutic intervention in patients who have CAD. (J Thorac Cardiovasc Surg 2015;150:240-6)

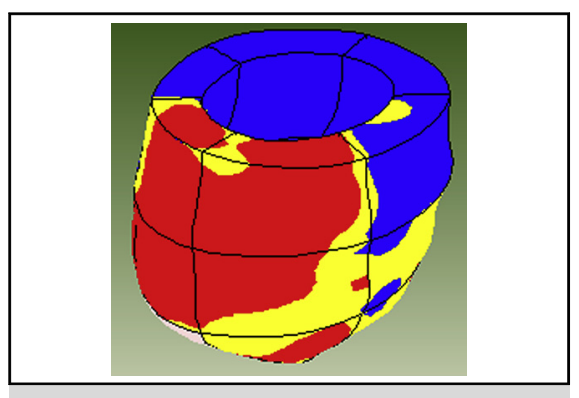

Magnetic resonance imaging-based, "normalized," regional contractile function in a patient with coronary artery disease.

\section{Central Message}

Normalized, high-resolution strain data quantify and localize regional contractile dysfunction in patients with coronary artery disease.

\section{Perspective}

This study confirms the clinical applicability and accuracy of MRI-based, normalized (by normal human strain database) LV multiparametric strain to quantify and regionally localize contractile dysfunction in patients with CAD. This high-resolution localization of regional wall motion abnormalities may help improve the accuracy of therapeutic intervention in these patients.

See Editorial Commentary page 247.
Patient-specific laboratory blood-test values fuel many clinical algorithms commonly utilized in the daily practice of evidence-based medicine. They are most often interpreted

From the Division of ${ }^{\mathrm{a}}$ Cardiothoracic Surgery, Department of Surgery; and ${ }^{\mathrm{b}}$ Cardiovascular Division, Department of Medicine, Washington University School of Medicine, St Louis, Mo.

This work was supported in part by funding from the National Institutes of Health, grants HL064869, HL069967, HL112084, and T32 HL007776.

Received for publication Feb 5, 2015; revisions received March 18, 2015; accepted for publication March 30, 2015; available ahead of print May 1, 2015.

Address for reprints: Michael K. Pasque, MD, Washington University School of Medicine, 660 South Euclid Ave, Campus Box 8234, St Louis, Missouri 63110 (E-mail: pasquem@wudosis.wustl.edu).

$0022-5223 / \$ 36.00$

Copyright (c) 2015 by The American Association for Thoracic Surgery http://dx.doi.org/10.1016/j.jtcvs.2015.03.049 by reference to large normal human databases, with "normal" ranges being defined by standard deviation (SD) from normal mean values. Therapeutic intervention is often triggered by patient-specific test values that fall $>2$ SDs from the mean. The comparison of an individual value to a normal database mean is known as "normalization." This process allows for nonsubjective interpretation of laboratory values that is easily incorporated into decision making in diagnostic and therapeutic clinical algorithms. Decision making in cardiac diagnostic and therapeutic algorithms unfortunately stands in stark contrast to this universally accepted medical paradigm.

Many factors determine branch-point decisions in clinical algorithms when coronary angiography demonstrates a significantly obstructive coronary arterial lesion. 


$$
\begin{aligned}
& \text { Abbreviations and Acronyms } \\
& \begin{aligned}
\text { CAD } & =\text { coronary artery disease } \\
\mathrm{ECG} & =\text { electrocardiography } \\
\mathrm{LV} & =\text { left ventricular } \\
\mathrm{MRI} & =\text { magnetic resonance imaging } \\
\mathrm{SPECT} & =\text { single-photon emission computed } \\
& \text { tomography }
\end{aligned}
\end{aligned}
$$

One such factor is the contractile function of the left ventricular (LV) region supplied by the diseased coronary artery. ${ }^{1,2}$ Clinicians most often make related decisions based on typewritten characterizations of LV contractile function, where "normalization" (comparison to the normal mean) most commonly is accomplished via observer visualization of nonquantified wall motion imaging, such as echocardiography. This nonquantitative, observer-dependent normalization has been repeatedly demonstrated to be inconsistent across time, observer, and imaging technique..$^{3-8}$

Heterogeneous LV contractile injury is expected in patients with coronary artery disease (CAD), with the distribution being dependent on specific coronary arterial atherosclerotic involvement. Somewhat surprisingly, our lab and others have additionally shown a consistent pattern of heterogeneous contractile injury in patients with dilated cardiomyopathy ${ }^{9-11}$ and aortic insufficiency. ${ }^{12}$ In other words, not only is normal human LV strain heterogeneous, ${ }^{13}$ but also, most LV strain injury occurs in a heterogeneous distribution. These studies establish the critical necessity of utilizing normalized strain metrics (by way of comparison to a normal human strain database $^{9,14}$ ) in the analysis of regional myocardial contractile function.

Our hypothesis is that the methodologies to quantify, subregionally localize, and database-normalize cardiac contractile data using high-resolution imaging are now available for application in the clinical setting. We further hypothesize that this normalized information is most applicable in patients with CAD and can be presented in an easily assimilated visual format that displays high-density, regionally varying contractile information utilizing intuitive, color-coded mapping over patientspecific 3-dimensional LV geometry.

\section{METHODS}

\section{Patient Characteristics}

The Human Research Protection Office at Washington University (St Louis, Mo) approved this study, and all subjects gave informed written consent. No gender-based or racial/ethnic-based exclusions were applied during patient recruitment.

Sixty patients with CAD (defined as having $\geq 1$ lesion of $>70 \%$ on coronary angiography) were enrolled in the study and underwent cardiac magnetic resonance imaging (MRI) with tissue tagging. An additional 60 healthy volunteers with no historical, physical, or clinical test evidence of any kind of heart disease also underwent cardiac MRI and contributed complete strain parameter information to a normal human strain database. Demographic data are presented in Table 1.

\section{Cardiac Magnetic Resonance Imaging}

All imaging studies were carried out using a 1.5T scanner (Siemens, Erlangen, Germany). Electrocardiogram-gated, short-axis, tagged MR images were acquired in multiple parallel planes, starting at the level of the mitral valve and extending to the apex of the left ventricle. Additionally, long-axis tagged images were acquired in 4 radially oriented planes separated by $45^{\circ}$ and intersecting at the approximate center of the LV cavity. In each imaging plane, a spatial modulation of magnetization radiofrequency tissue-tagging preparation was applied, followed by a 2-dimensional, balanced, steady-state free precession cine image acquisition. $^{15,16}$ This process creates markers on the myocardium that deform with the tissue, which allows for quantification of wall motion. Typical imaging parameters were: repetition time of 32.4 milliseconds, echo time of 1.52 milliseconds, field of view of $350 \times 350 \mathrm{~mm}$, flip angle of $20^{\circ}$, tag spacing of $8 \mathrm{~mm}$, and slice thickness of $8 \mathrm{~mm}$.

\section{Strain Analysis}

Strain measurements were obtained for all study participants using an established and validated method developed at our institution. ${ }^{17-20}$ Clinical validation of this methodology has demonstrated resistance to interobserver variability. ${ }^{21,22}$ Briefly, endocardial and epicardial wall boundaries were manually identified on each of the tagged images used in the study. Tag lines were tracked on each of the images semi-automatically, utilizing an active contour approach. ${ }^{17}$

Three-dimensional displacements were computed for the systolic interval by tracking the intramyocardial intersection points of the 3 tag surfaces. The end-diastolic wall boundaries were used to create a finite element model of the left ventricle. Using the anterior and posterior attachment points of the right ventricle to the left ventricle as landmarks, a standardized 18-element mesh was created for each model. A leastsquares fitting of the displacement data was used to obtain a continuous description of displacement for the entire model.

Using the results of this fitting, circumferential strain, longitudinal strain, and the minimum principal strain angle were computed over a standardized grid of 15,300 points for the entire left ventricle. The minimum principal strain angle is the angle between the plane formed by the circumferential and longitudinal axes and the axis of minimum principal strain. ${ }^{23}$ In the left ventricle, this angle quantifies the orientation of maximal contraction. The finite element software package StressCheck (ESRD, Inc, St Louis, Mo) was used to obtain the strain measurements for this study.

\section{Magnetic Resonance Imaging-Based \\ Multiparametric Strain Z-Score Analysis}

A total of 120 test subjects were subjected to full strain analysis after cardiac MRI with radiofrequency tissue-tagging. In all individuals, 3-dimentional LV systolic strain components were calculated from tag surface intersection displacement data over a standardized grid of 15,300 LV points. A normal human strain database was constructed using strain measurements from 60 healthy volunteers. At each LV grid point, and for each strain metric, measurements from the healthy volunteers were used to generate a normal mean and SD at that particular point. This database of normal strain values allows for patient-specific, pointspecific, and parameter-specific comparisons and z-score generation.

A z-score is a statistical measurement that relates an individual score to a mean in a group of scores. The value of the $\mathrm{z}$-score is directly proportional to its variation from the mean. A value of 0 means the score is the same as the mean, and a value of 1 is $1 \mathrm{SD}$ above the mean. 
TABLE 1. Characteristics of patients with CAD

\begin{tabular}{lcc}
\hline \multicolumn{1}{c}{ Characteristic } & Patients with CAD & Healthy volunteers \\
\hline Age (y), mean \pm SD & $60.7 \pm 9.1$ & $33.1 \pm 10.8$ \\
Gender, female & $20(12 / 60)$ & $53(32 / 60)$ \\
Diabetes & $28(14 / 50)$ & 0 \\
Hypertension & $84(42 / 50)$ & 0 \\
COPD & $6(3 / 50)$ & 0 \\
Chronic renal insufficiency & $32(16 / 50)$ & 0 \\
Atrial fibrillation & $16(8 / 50)$ & 0 \\
NYHA class I & $54(27 / 50)$ & $100(60 / 60)$ \\
NYHA class II & $34(17 / 50)$ & 0 \\
NYHA class III & $12(6 / 50)$ & 0 \\
Previous CABG & $32(16 / 50)$ & 0 \\
Echocardiographic ejection & $42 \pm 12$ & NA \\
fraction $(\%)$ & & \\
\hline
\end{tabular}

Values are $\%$ (n of total), unless otherwise indicated. $C A D$, Coronary artery disease; $\mathrm{SD}$, standard deviation; $C O P D$, chronic obstructive pulmonary disorder; $N Y H A$, New York Heart Association; $C A B G$, coronary artery bypass grafting; $N A$, not applicable.

A second group of 60 patients with known CAD underwent cardiac MRI, and z-scores for 3 strain parameters at each of the $15,300 \mathrm{LV}$ grid-points were calculated by reference to the normal human strain database. The z-scores for 3 strain metrics at each point were then averaged to generate a single multiparametric strain $z$-score value at each of the 15,300 points.

Blinded clinicians with no knowledge of the MRI-based multiparametric strain results performed an extensive review of all available medical records for every patient with CAD included in our study population. The goal in this clinical review was to identify myocardial regions that had sustained myocardial infarction, via echocardiography, single-photon emission computed tomography (SPECT), or electrocardiography (ECG), because this would confirm that an LV region had significant contractile dysfunction, by which the accuracy of our strain methodology could be tested. Using gold-standard definitions (Q-waves on ECG, infarction on radionuclide SPECT, and/or akinesis or dyskinesis on echocardiography), in each $\mathrm{CAD}$ patient, regional wall motion abnormalities were identified.

Once these data were compiled, the $\mathrm{z}$-scores were examined in each of the regions identified by the clinical review. If the average $\mathrm{z}$-score was $>1.5$ for the affected region, then for that particular gold-standard imaging modality, the multiparametric strain analysis was considered accurate. Accurate identification of affected regions was assessed for each imaging modality and compared.

\section{RESULTS}

Multiparametric strain information was successfully obtained in all 60 patients with $\mathrm{CAD}$, with a postprocessing display of "normalized" microregional contractile function on patient-specific LV geometry. The display of "normalized" microregional contractile function by intuitive $\mathrm{z}$-score color contour mapping for all 60 patients with CAD is represented in Figure 1. For ease of display, only a single view of each patient-specific, rotatable, semitransparent 3-dimensional model is shown.

Further, to display the functionality and applicability of this methodology in a paradigm with which most clinicians are familiar, we scaled the color contouring based on thresholds similar to those utilized in the clinical integration of patient laboratory blood-test values. Specifically, those $<1 \mathrm{SD}$ from the normal mean are colored blue; 1 to 2 SDs are yellow; and $>2$ SDs are red. For comparison, a healthy volunteer with normal contractile function would appear diffusely blue (Figure 2). The average subregional z-score data from the 60 patients with CAD are displayed in Table 2 and suggest that we will more accurately define clinically relevant thresholds with the accrual of more clinical data. This patient-specific normalized microregional contractile function is mapped (Figure 1) over patient-specific 3-dimensional LV geometry as defined by individual patient MRI-based epicardial and endocardial wall boundaries.

A blinded chart review of the 60 patients with known CAD revealed that $55 \%$ (33 of 60 ) of these patients had evidence of regional wall motion abnormalities, as determined by current clinical gold-standard diagnostic criteria (Q-waves on ECG, infarction on radionuclide SPECT, and/or akinesis or dyskinesis on echocardiogram). In this population, abnormalities were found in the anterior wall in $66 \%$ ( 22 of 33 ) of patients, the anterolateral wall in $39 \%$ (13 of 33) of patients, anteroseptal in 58\% (19 of 33), and posteroseptal, posterior, and posterolateral in $88 \%(29$ of 33) of patients. In most patients, the so-called gold-standard criteria were inconsistent regarding the presence or absence of wall motion abnormalities. In fact, consistency between all gold-standard metrics was found in only 19\% (6 of 31) of the patients with CAD for whom $\geq 2$ gold-standard metrics were available.

Based on our initial use of MRI-based multiparametric strain analysis in a small test group of CAD patients with known regional nonviability, ${ }^{14}$ a subregional average $\mathrm{z}$-score of $>1.5$ was used as the threshold indicative of clinically significant contractile dysfunction. Of the patients in our complete study group who had wall motion abnormalities on ECG, SPECT, and echocardiogrpahy, the blinded MRI-based multiparametric strain z-score localization of these contractile abnormalities using this z-score threshold was accurate in $89 \%, 97 \%$, and $95 \%$, respectively. An example of SPECT compared with the MRI-based, normalized stain analysis in a patient with $\mathrm{CAD}$ is represented in Figure 3.

\section{DISCUSSION}

Magnetic resonance imaging-based multiparametric strain analysis provides a high-resolution quantification of microregional LV contractile function. ${ }^{14,24}$ Patientspecific 3-dimensional intramural LV point displacements obtained using MRI tissue-tagging are used to quantify each of 3 strain parameters (circumferential magnitude, longitudinal magnitude, and minimum principal strain angle $^{23}$ ) at each of $15,300 \mathrm{LV}$ grid-points. A z-score is calculated for each patient-specific raw strain parameter value at each grid-point by its comparison to the normal 


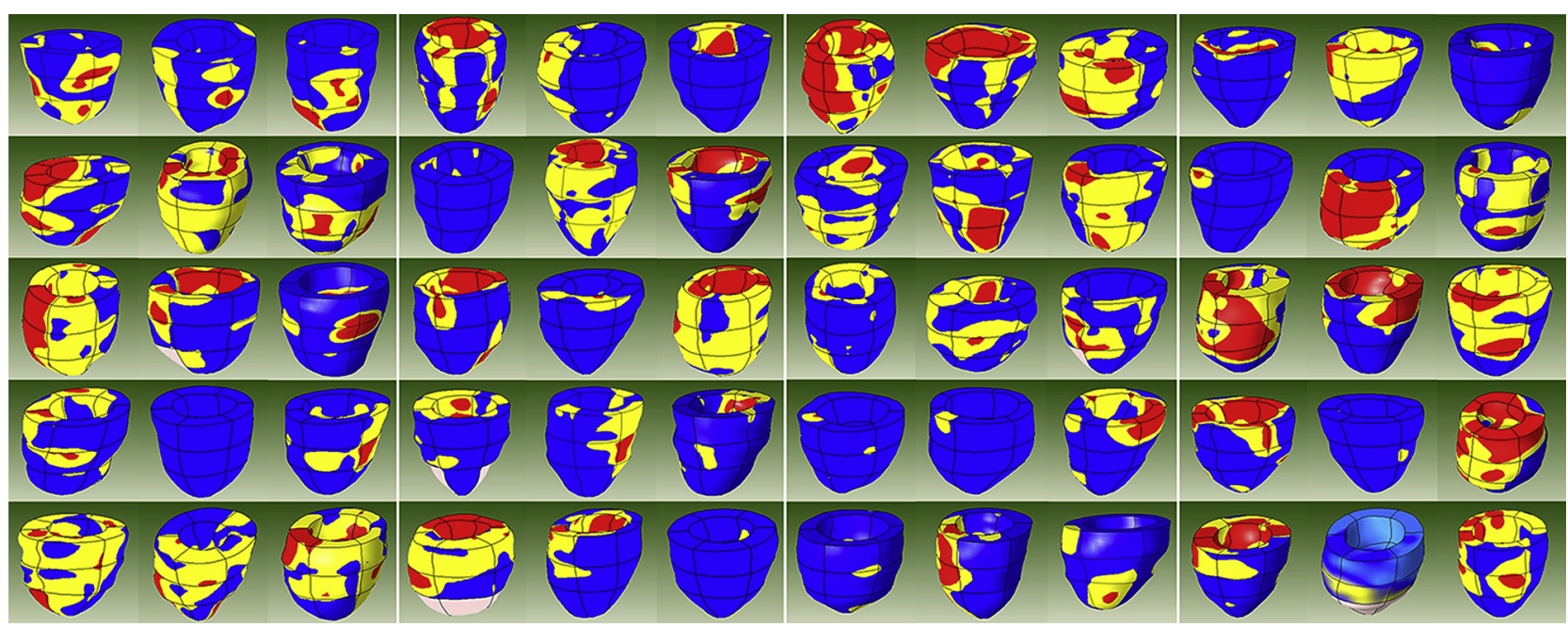

FIGURE 1. The display of "normalized," microregional contractile function by intuitive z-score color contour mapping $(<1 \mathrm{SD}=$ blue $; 1-2 \mathrm{SDs}=$ ellow; $>2$ SDs $=r e d$ ) over patient-specific 3D left ventricular geometry for all 60 patients with coronary artery disease. The resolution of each MRI image is 72 pixels/inch; the 3D color contour maps are 300 pixels/inch.

mean and SD (normal human strain database) for that specific strain parameter at that precise LV grid-point.

Thus, completion of a single routine study of patients with $\mathrm{CAD}$, utilizing a standard 15,300 grid-point model, requires 45,900 microprocessor-controlled database comparisons. This normalization process allows multiple individually effective strain parameters (with variable data ranges) to be combined into more-powerful composite multiparametric strain indices. ${ }^{14,25}$ These multiparametric strain values, which represent a highly quantified normalization of point-specific LV contractile function, are plotted over patient-specific LV geometry with color contour mapping to enhance intuitive interpretation.

To be clinically applicable, the capabilities of MRI-based multiparametric strain analysis must be demonstrated in the actual patient populations and clinical settings in which it may have diagnostic or therapeutic value. We therefore report the results of its application in a study group of 60 patients who have known ischemic CAD and, therefore, a high incidence of regional contractile dysfunction. This testing has confirmed not only its accuracy in detecting regional wall motion abnormalities, but also the applicability of this high-resolution, dynamic cardiac geometric data acquisition in the clinical setting frequented by patients with advanced atherosclerotic coronary occlusive disease. We have, in fact, already applied this same methodology effectively in multiple other patient populations, including those with aortic valvular disease, ${ }^{12,26}$ mitral valvular disease, ${ }^{27}$ and nonischemic dilated cardiomyopathy. ${ }^{9}$

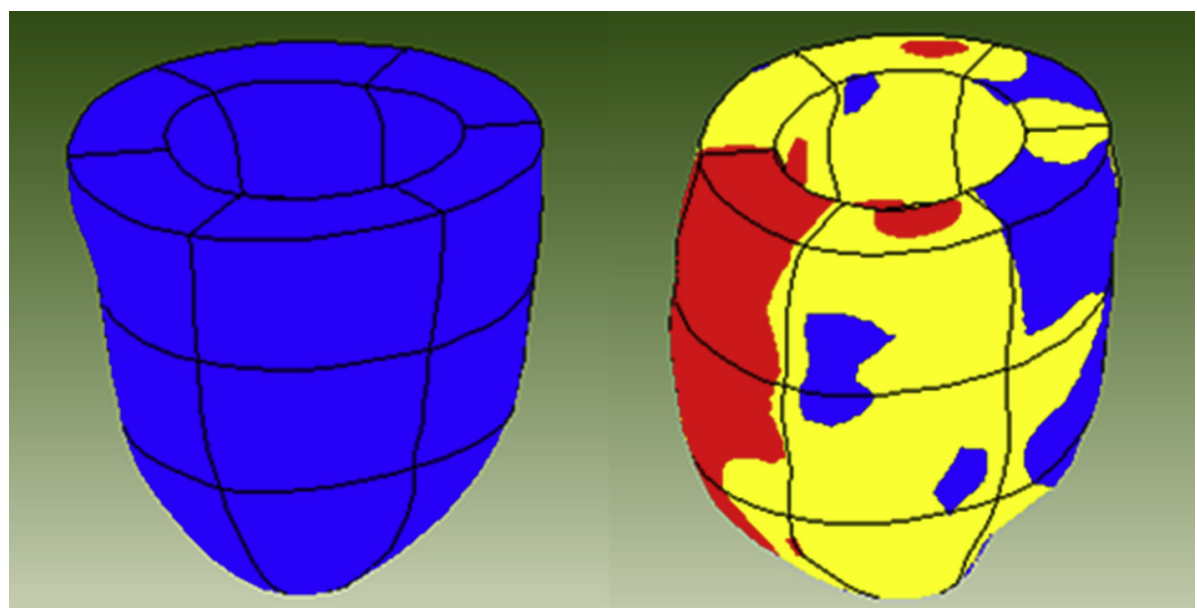

FIGURE 2. The display of "normalized," microregional contractile function by z-score color contour mapping in a healthy volunteer (left) is contrasted with that of a patient (right) with severe, regional, contractile impairment secondary to myocardial infarction $(<1 \mathrm{SD}=$ blue; $1-2 \mathrm{SDs}=$ yellow; $>2$ $\mathrm{SDs}=r e d)$. 
TABLE 2. Regional average MRI-based multiparametric strain z-scores from 60 patients

\begin{tabular}{lccccrr}
\hline & Posteroseptal & Anteroseptal & Apical & Anterolateral & Posterolateral & Posterior \\
\hline Base & $1.13 \pm 1.01$ & $1.43 \pm 1.04$ & $1.07 \pm 0.72$ & $0.88 \pm 0.80$ & $1.05 \pm 1.07$ & $1.14 \pm 1.08$ \\
Midventricle & $1.39 \pm 1.35$ & $1.34 \pm 1.22$ & $1.20 \pm 0.95$ & $1.07 \pm 0.87$ & $1.45 \pm 1.15$ & $1.42 \pm 1.07$ \\
Apex & $0.97 \pm 0.96$ & $0.87 \pm 0.88$ & $0.79 \pm 0.75$ & $0.77 \pm 0.81$ & $0.97 \pm 0.90$ & $0.96 \pm 0.80$ \\
\hline
\end{tabular}

The patient-specific LV contractile information supplied by this methodology is the product of complex postprocessing of a substantial volume of quantified microregional, myocardial point-displacement data. The data resulting

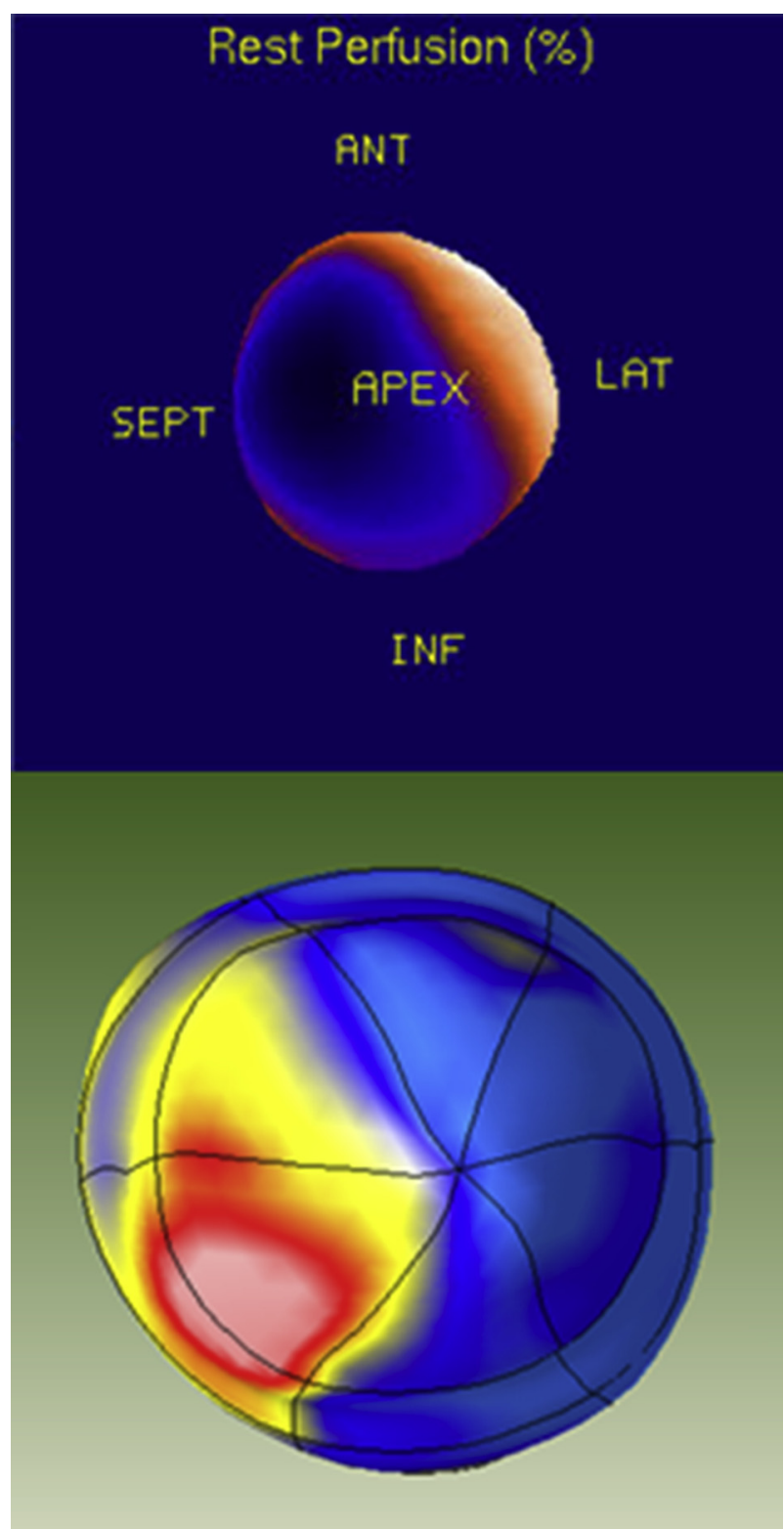

FIGURE 3. Comparison of a SPECT image (top) to MRI-based, "normalized," microregional contractile function (bottom) in the same patient with coronary artery disease. ANT, Anterior; SEPT, septal; $L A T$, lateral; $I N F$, inferior. from the previously described 45,900 microprocessorcontrolled database comparisons alone would overwhelm even the most ardent supporter of this analysis. In the past, these scrolls of typewritten mathematical data were precisely what made this sort of complex microregional contractile analysis irrelevant to the clinician, who had neither the time nor expertise to decipher the information.

For this complex microregional contractile information to be clinically relevant-even in the age of the electronic medical record (EMR) - it must be presented in a 3dimensional color contour visual format that allows easy and immediate clinical assimilation of "normalized," microregional, LV contractile information in relation to patient-specific LV geometry. Our further contention is that the patient-specific, regional, LV contractile data that fuel cardiac clinical algorithms can be presented in a normalized $\mathrm{z}$-score format similar to that which is already so familiar to physicians in the interpretation of patientspecific, laboratory blood-test values.

Traditionally, echocardiography most often supplies the dynamic LV geometric input on which clinical decisions for patients with CAD are made. Observer-dependent visual differentiation into 1 of 4 contractile classifications (normal, hypokinetic, akinetic, dyskinetic) supplies only a gross normalization across 6 arbitrarily assigned geometric LV regions. Thus, unlike the quantified normalization commonly applied to laboratory blood testing, regional contractile function is qualitative, not normalized, and subject to interobserver variation. Interpreter and temporal inconsistency is predictable, because the assessment of regional contractile function is most often dependent on the visual detection of 1-mm LV wall-thickness changes in 8-mm thick walls that are moving in and out of the echocardiographic imaging plane with every breath and LV contraction. ${ }^{7}$ In contrast, our methodology relies heavily on a quantitative comparison of a patient-specific strain to a fixed standard (normal human strain database), allowing very little tolerance for temporal inconsistency. ${ }^{21,22}$

Several other alternatives to standard 2-dimensional echocardiography have been evaluated. Speckle-tracking echocardiography, which is based on the spatial displacement of "speckles" generated by the interaction between ultrasound beams and myocardial fibers, ${ }^{28}$ was designed to provide further information on global and regional contractile function. This modality tracks these speckles in 3 dimensions, during the cardiac cycle, allowing for more detailed information on myocardial contractility. 
Despite improvement in quantifying LV dysfunction, this imaging modality is still constrained by limited imaging windows, low-resolution image quality, and significant user-dependent interobserver variability. ${ }^{29}$ Three-dimensional echocardiography provides higherresolution and more-detailed images than conventional echocardiography, but it is still limited by subjective interpretation, variable acoustic windows, and interobserver variability. ${ }^{30}$ In contrast, MRI-based multiparametric strain z-score "normalization" is based on fully 3-dimensional, high-resolution imaging evaluated in a highly quantified, nonsubjective manner.

One of the obvious and long-awaited advantages of the EMR is the ability it allows to present large volumes of complex mathematical patient data, such as that obtained from MRI-based multiparametric strain z-score analysis, in an easy-to-interpret, intuitive, visual format. The addition of quantified normalization to a visually intuitive format maximizes the efficient assimilation of this information, even by physicians who do not have subspecialty training in cardiology, radiology, or cardiothoracic surgery. In fact, given that the primary determination of "normal versus abnormal" is already automatically made by nonbiased, repeatedly consistent computational methods, and then presented in an intuitive format, the results can be easily interpreted by not only cardiac imaging subspecialists, but also primary care physicians. In addition, the presentation of regional contractile data in an already normalized format allows microregions with contractile function falling outside of 1 or 2 SDs to be clearly delineated (yellow and red in our images) to any interested party, including patients themselves.

The presentation of complex medical information in such a format represents the zenith of the EMR allowing even patients to gain an improved understanding of their disease process. This approach is the clear future of medicine with patients increasingly being asked to participate as informed consumers and assume more responsibility in their medical decision making. The EMR provides the ideal mechanism to present and explain complex medical information-in this case, patient-specific, microregional, LV contractile information-in a format that can be readily understood by patients themselves.

As the EMR matures, this visual presentation of microregional contractile information will take full advantage of the obvious benefits the EMR offers. A laptop computer or touchpad is all that is necessary to spin a 3-dimensional LV model of the patient's very own normalized contractile function, projected on an accurate model of their LV geometry. This type of usage has considerable potential for immediate impact in the clinical application of this methodology in directing therapeutic intervention in those patients with a regionally variable degree of ischemic contractile injury.
The proposed imaging modality has several important limitations. In particular, MRI can be expensive, and postprocessing of myocardial strain can be time consuming. However, with advances in MR image acquisition technology and more-automated data analysis, our current scan times are $<30$ minutes, with associated data analysis requiring $<20$ minutes.

Another limitation of this methodology, common to other measurements of regional myocardial contractility, is that it can quantify only myocardial strain. Ventricular contractility takes into account strain (how well the wall is moving), stress (how much force the wall is exerting), and time (how quickly the wall is moving). Ideally, stress and strain would be concurrently quantified. Unfortunately, current methods for accurately quantifying regional myocardial stress in the clinical setting are severely limited. Finally, the average age of our healthy volunteers is significantly lower than that in the CAD study group. Nonetheless, investigations into age-related differences in strain suggest that significant differences occur only at the extreme ends of the normal population age range. ${ }^{31}$

This investigation has further confirmed the limited ability of current clinical gold-standard contractile metrics to consistently and quantitatively localize regional LV contractile abnormalities. As a reasonable clinical alternative, MRI-based multiparametric strain 3-dimensional z-score "normalization" of microregional contractile function, by comparison of patient-specific raw strain values to a normal human strain database, can accurately quantify, normalize, and localize LV contractile dysfunction on a microregional basis. With the widespread implementation of electronic medical records, this intuitive 3-dimensional color contour mapping of normalized, regional, contractile function on patient-specific 3-dimensional LV geometry has considerable potential to replace the current nonquantitative, typewritten reports on which critical clinical decisions are often based.

\section{Conflict of Interest Statement}

Michael K. Pasque, MD, Brian P. Cupps, PhD, and Washington University may receive income based on a license for related technology, issued by the university to CardioWise, Inc. CardioWise, Inc did not support this work. All other authors have nothing to disclose regarding commercial support.

The authors appreciate the contributions of Dr Andrew Kates and Dr Jennifer Lawton.

\section{References}

1. Nishimura RA, Otto CM, Bonow RO, Carabello BA, Erwin JP III, Guyton RA, et al. 2014 AHA/ACC guideline for the management of patients with valvular heart disease: executive summary: a report of the American College of Cardiology/American Heart Association Task Force on Practice Guidelines. J Am Coll Cardiol. 2014;63:2438-88. 
2. Amsterdam EA, Wenger NK, Brindis RG, Casey DE Jr, Ganiats TG, Holmes DR Jr, et al. 2014 AHA/ACC Guideline for the Management of Patients With Non-ST-Elevation Acute Coronary Syndromes: A Report of the American College of Cardiology/American Heart Association Task Force on Practice Guidelines. J Am Coll Cardiol. 2014;64:e139-228.

3. Vermes E, Guyon P, Weingrod M, Otmani A, Soussana C, Halphen C, et al. Assessment of left ventricular regional wall motion by color kinesis technique: comparison with angiographic findings. Echocardiography. 2000; 17:521-7.

4. Peart I, Austin A, Hall RJ. Subjective analysis of cross-sectional echocardiograms: reproducibility and sources of variability. Eur Heart J. 1987;8:171-8.

5. Badano L, Stoian J, Cervesato E, Bosimini E, Gentile F, Giannuzzi P, et al. Reproducibility of wall motion score and its correlation with left ventricular ejection fraction in patients with acute myocardial infarction. Am J Cardiol. 1996;78:855-8.

6. Blondheim DS, Beeri R, Feinberg MS, Vaturi M, Shimoni S, Fehske W, et al. Reliability of visual assessment of global and segmental left ventricular function: a multicenter study by the Israeli Echocardiography Research Group. J Am Soc Echocardiogr. 2010;23:258-64.

7. Kvitting JP, Wigstrom L, Strotmann JM, Sutherland GR. How accurate is visual assessment of synchronicity in myocardial motion? An in vitro study with computer-simulated regional delay in myocardial motion: clinical implications for rest and stress echocardiography studies. J Am Soc Echocardiogr. 1999;12: 698-705.

8. Picano E, Lattanzi F, Orlandini A, Marini C, L'Abbate A. Stress echocardiography and the human factor: the importance of being expert. J Am Coll Cardiol. 1991;17:666-9.

9. Joseph S, Moazami N, Cupps BP, Howells A, Craddock H, Ewald G, et al. Magnetic resonance imaging-based multiparametric systolic strain analysis and regional contractile heterogeneity in patients with dilated cardiomyopathy. J Heart Lung Transplant. 2009;28:388-94.

10. Potter DD, Araoz PA, Ng LL, Kruger DG, Thompson JL III, Hamner CE, et al. Cardiotropin-1 and myocardial strain change heterogeneously in cardiomyopathy. J Surg Res. 2007;141:277-83.

11. Young AA, Dokos S, Powell KA, Sturm B, McCulloch AD, Starling RC, et al. Regional heterogeneity of function in nonischemic dilated cardiomyopathy. Cardiovasc Res. 2001:49:308-18.

12. Knutsen AK, Ma N, Taggar AK, Brady BD, Cupps BP, Pasque MK. Heterogeneous distribution of left ventricular contractile injury in chronic aortic insufficiency. Ann Thorac Surg. 2012;93:1121-7.

13. Fonseca CG, Oxenham HC, Cowan BR, Occleshaw CJ, Young AA. Aging alters patterns of regional nonuniformity in LV strain relaxation: a 3-D MR tissue tagging study. Am J Physiol Heart Circ Physiol. 2003;285: H621-30.

14. Cupps BP, Bree DR, Wollmuth JR, Howells AC, Voeller RK, Rogers JG, et al. Myocardial viability mapping by magnetic resonance-based multiparametric systolic strain analysis. Ann Thorac Surg. 2008;86:1546-53.

15. Axel L, Dougherty L. Heart wall motion: improved method of spatial modulation of magnetization for MR imaging. Radiology. 1989;172:349-50.

16. Axel L, Dougherty L. MR imaging of motion with spatial modulation of magnetization. Radiology. 1989;171:841-5.
17. Moulton MJ, Creswell LL, Downing SW, Actis RL, Szabo BA, Vannier MW, et al. Spline surface interpolation for calculating 3-D ventricular strains from MRI tissue tagging. Am J Physiol. 1996;270:H281-97.

18. Moustakidis P, Cupps BP, Pomerantz BJ, Scheri RP, Maniar HS, Kates AM, et al Noninvasive, quantitative assessment of left ventricular function in ischemic cardiomyopathy. J Surg Res. 2004;116:187-96.

19. Pirolo JS, Creswell LL, Bresina SJ, Perman WH, Szabo BA, Myers KW, et al. Regional myocardial stress distribution from magnetic resonance image-based mathematical models. Ann Thorac Surg. 1991;52:276-84.

20. Pirolo JS, Bresina SJ, Creswell LL, Myers KW, Szabo BA, VAnnier MW, et al. Mathematical three-dimensional solid modeling of biventricular geometry. Ann Biomed Eng. 1993;21:199-219.

21. Kar J, Knutsen AK, Cupps BP, Zhong X, Pasque MK. Three-dimensional regional strain computation method with displacement encoding with stimulated echoes (DENSE) in non-ischemic, non-valvular dilated cardiomyopathy patients and healthy subjects validated by tagged MRI. J Magn Reson Imaging. 2015;41:386-96.

22. Kar J, Knutsen AK, Cupps BP, Pasque MK. A validation of two-dimensional in vivo regional strain computed from displacement encoding with stimulated echoes (DENSE), in reference to tagged magnetic resonance imaging and studies in repeatability. Ann Biomed Eng. 2014;42:541-54.

23. Cupps BP, Pomerantz BJ, Krock MD, Villard J, Rogers J, Moazami N, et al. Principal strain orientation in the normal human left ventricle. Ann Thorac Surg. 2005;79:1338-43.

24. Cupps BP, Taggar AK, Reynolds LM, Lawton JS, Pasque MK. Regional myocardial contractile function: multiparametric strain mapping. Interact Cardiovasc Thorac Surg. 2010;10:953-7.

25. Bree D, Wollmuth JR, Cupps BP, Krock MD, Howells A, Rogers J, et al. Low-dose dobutamine tissue-tagged magnetic resonance imaging with 3-dimensional strain analysis allows assessment of myocardial viability in patients with ischemic cardiomyopathy. Circulation. 2006;114:I33-6.

26. Brady BD, Knutsen AK, Ma N, Gardner R, Taggar AK, Cupps BP, et al. MRI-based multiparametric strain analysis predicts contractile recovery after aortic valve replacement for aortic insufficiency. J Card Surg. 2012;27:415-22.

27. Maniar HS, Brady BD, Lee U, Cupps BP, Kar J, Wallace KM, et al. Early left ventricular regional contractile impairment in chronic mitral regurgitation occurs in a consistent, heterogeneous pattern. J Thorac Cardiovasc Surg. 2014;148: 1694-9.

28. Mondillo S, Galderisi M, Mele D, Cameli M, Lomoriello VS, Zaca V, et al. Speckle-tracking echocardiography: a new technique for assessing myocardial function. J Ultrasound Med. 2011;30:71-83.

29. Leischik R, Dworrak B, Hensel KO. Intraobserver and interobserver reproducibility for radial, circumferential and longitudinal strain echocardiography. Open Cardiovasc Med J. 2014;8:102-9.

30. Soliman OI, Kirschbaum SW, van Dalen BM, van der Zwaan HB, Mahdavian Delavary B, Vletter WB, et al. Accuracy and reproducibility of quantitation of left ventricular function by real-time three-dimensional echocardiography versus cardiac magnetic resonance. Am J Cardiol. 2008;102:778-83.

31. Kaku K, Takeuchi M, Tsang W, Takigiku K, Yasukochi S, Patel AR, et al. Agerelated normal range of left ventricular strain and torsion using three-dimensional speckle-tracking echocardiography. J Am Soc Echocardiogr. 2014;27:55-64.

Key Words: MRI, coronary artery disease, contractility

Readers who found these articles interesting may also like to read the following papers found in recent and future issues of our sister publications, Seminars in Thoracic and Cardiovascular Surgery and Operative Techniques in Thoracic and Cardiovascular Surgery!

Evolving Technology/Basic Science

State of the Art: RichardWeisel. The Promise and Challenges of Cardiac Stem Cell Therapy. Semin Thorac Cardiovasc Surg. Spring 2014;26(1):44-52. 